

\title{
Evaluación de la severidad de Sigatoka negra (Mycosphaerella fijiensis Morelet) en plátano "Barraganete" bajo fertilización con magnesio
}

\author{
José Randy Cedeño-Zambrano ${ }^{1} \mathbb{D}$, Eduar Josué Díaz-Barrios $^{2} \mathbb{D}$, Eder de Jesús Conde- \\ López $^{3}{ }^{\mathbb{D}}$, Abrahan Rodolfo Cervantes-Álava ${ }^{4}$ (D) Leonardo Enrique Avellán-Vásquez ${ }^{5}$ (D) \\ Myriam Elizabeth Zambrano-Mendoza ${ }^{6}{ }^{\mathbb{D}}$, Juan Pablo Tobar-Galvéz ${ }^{7} \mathbb{D}^{\mathbb{D}}$, Sandra Tatianá \\ Estévez-Chica $^{7}$ (D) Adriana Beatriz Sánchez-Urdaneta ${ }^{8^{*}}$ (D)
}

${ }^{1}$ Carrera de Ingeniería Agropecuaria, Universidad Laica Eloy Alfaro de Manabí, Extensión en El Carmen. Manabí, Ecuador. Estudiante de Doctorado de Ciencias Agrarias, Facultad de Agronomía, Universidad del Zulia, Venezuela. ${ }^{2}$ Ing. Agr., Docente Unidad Educativa Maranatha, El Carmen, Manabí, Ecuador. ${ }^{3}$ Ing. Agr. Libre Ejercicio de la Profesión. Venezuela. ${ }^{4}$ Facultad de Ciencias Agropecuarias, Universidad Técnica de Machala, Machala, Ecuador. Estudiante de Doctorado de Ciencias Agrarias, Facultad de Agronomía, Universidad del Zulia, Venezuela. ${ }^{5}$ Carrera de Ingeniería Agropecuaria, Universidad Laica Eloy Alfaro de Manabí, Extensión en El Carmen. Manabí, Ecuador. Estudiante de Doctorado de Ingeniería Agraria, Alimentaria, Forestal y de Desarrollo Rural Sostenible, Universidad de Córdoba, España. ${ }^{6}$ Carrera de Ingeniería Agropecuaria, Universidad Laica Eloy Alfaro de Manabí, Extensión en El Carmen. Manabí, Ecuador. ${ }^{7}$ Investigadores Independientes, Manabí, Ecuador. ${ }^{8}$ Departamento de Botánica, Facultad de Agronomía, Universidad del Zulia. Maracaibo, Venezuela.

*Autor de correspondencia: usanchez@fa.luz.edu.ve https://doi.org/10.22209/rt.v44n1a01

Recepción: 27 de febrero de 2020 | Aceptación: 20 de agosto de 2020 | Publicación: 01 de enero de 2021

\section{Resumen}

Sigatoka negra (SN) es la enfermedad foliar que representa la principal limitante en la producción de plátano a nivel mundial. Por lo que, la presente investigación tuvo como objetivo evaluar la severidad de Sigatoka negra (Mycosphaerella fijiensis Morelet) en plátano "Barraganete" bajo fertilización con magnesio, en El Carmen, Ecuador. Se utilizó un diseño de bloques completamente al azar con tres repeticiones; con 288 plantas sembradas a una densidad de 2.222 plantas/ ha. Se realizó una fertilización básica de N-P-K (100-40-150 Kg/ha), con seis dosis de Mg0 (0, 25, 50, 75, 100 y 125 Kg/ ha), fraccionada en tres partes (12, 18 y 24 hojas). Semanalmente se inspeccionaron las hojas 3 , 4 y 5 con la escala de Fouré, analizando estos datos mediante la metodología de medidas repetidas en el tiempo. Para evaluar la incidencia de SN semanalmente se inspeccionaron las hojas 3, 4 y 5 con la escala de Fouré, junto con deshoje y cirugía. Se evaluaron 10 plantas por tratamiento de fertilización, se realizaron seis aplicaciones de fungicidas con productos de contacto y sistémicos. Durante la semana 20 en la hoja 3 se presentó la mayor severidad de SN, inclusive fue superior al nivel severo en los tratamientos de 75 y $125 \mathrm{Kg} / \mathrm{ha}$ de MgO. Las ecuaciones de los modelos polinómicos determinaron que con la dosis de $25 \mathrm{Kg} / \mathrm{ha}$ de MgO, se obtuvo la menor severidad de SN. Conocer las condiciones ambientales y supervisión permite realizar un mejor manejo agronómico.

Palabras clave: Musa sp.; Mycosphaerella fijiensis; plátano “Barraganete”; manejo del cultivo; fertilización.

\section{Evaluation of the severity of Black Sigatoka (Mycosphaerella fijiensis Morelet) in plantain 'Barraganete' under magnesium fertilization}

\begin{abstract}
Black Sigatoka (BS) is a foliar disease that represents the main limiting factor in plantain production worldwide. Therefore, the research aimed to evaluate the severity of Black Sigatoka (Mycosphaerella fijiensis Morelet) in 'Barraganete'
\end{abstract}


plantain under magnesium fertilization, in El Carmen, Ecuador. A completely random block design with three repetitions was used; with 288 seeded plants with adensity of 2,222 plants/ha. Basic fertilization of N-P-K (100-40-150 Kg/ha) was conducted with six doses of $\mathrm{MgO}(0,25,50,75,100$, and $125 \mathrm{Kg} / \mathrm{ha})$, segmented into three parts (12, 18 and 24 leaves). Every week, leaf 3, 4, and 5 were examined with the Fouré scale, these data were analyzed by using the methodology of repeated measures over time. To assess the incidence of BS weekly, leaf 3, 4, and 5 were inspected with the Fouré scale, along with leaf removal and surgery. Ten plants per fertilization treatment were evaluated, and six fungicide applications were performed with contact and systemic products. The highest severity of BS was shown for all the treatments in week 20, on leaf 3, reaching levels higher than severe in treatments of 75 and $125 \mathrm{Kg} / \mathrm{ha}$ of $\mathrm{MgO}$. The equations of the polynomial models determined that with the dose of $25 \mathrm{Kg} / \mathrm{ha}$ of $\mathrm{MgO}$ the lowest severity of BS was observed. Knowing the environmental conditions and supervision allow for better agronomic management.

Keywords: Musa sp.; Mycosphaerella fijiensis; plantain 'Barraganete'; crop management; fertilization.

\section{Introducción}

Entre los cultivos de frutos tropicales destacan los plátanos y los bananos, no solo por los volúmenes producidos, sino también en términos de satisfacer las necesidades calóricas de millones de personas, especialmente en África, Asia y América; además de los frutos, se conoce que cualquier otra parte de la planta posee valor comercial o medicinal [1].

Sigatoka negra (SN, Mycosphaerella fijiensis Morelet), es uno de los patógenos que mayor impacto económico, social y ambiental ha tenido en la historia de la agricultura, y particularmente en la producción mundial de plátanos [2]. Es la principal enfermedad foliar en términos económicos de la producción de plátanos y bananos en el mundo [3], ya que puede reducir en un $50 \%$ los rendimientos $[4,5]$. Los costos de manejo de la enfermedad en plantaciones comerciales oscilan en el $27 \%$ de los costos totales de producción [6].

El principal efecto de la enfermedad es la prolongación del periodo de floración a cosecha y la aceleración del proceso de maduración del fruto en la planta y después de cosechado, bajo condiciones de control deficiente, las pérdidas de mayor magnitud se relacionan con la eliminación de racimos en el campo, provenientes de plantas con poca cantidad de hojas (menos de cuatro hojas sanas), debido al riesgo de maduración prematura del fruto ocasionado por la enfermedad; por ello, se deben mantener, al momento de la floración, un mínimo de ocho hojas funcionales, lo anterior, indica la importancia del manejo agronómico para llegar a la floración, por lo menos, con ocho hojas con bajo grado de severidad de sigatoka negra $[7,8]$.

El magnesio $(\mathrm{Mg})$ es el segundo catión más abundante en las plantas, está involucrado en diversos procesos fisiológicos y bioquímicos (fotosíntesis, activación enzimática y síntesis de ácidos nucleicos y proteínas). Su deficiencia no solo afecta la productividad y la calidad de los cultivos,si no que también se comporta como un elemento antagónico en el suelo del K y el Ca [9, 10].

La aplicación excesiva de fertilizantes como $\mathrm{K}$ y $\mathrm{NH}_{4}{ }^{+}$interfieren antagónicamente con la absorción de Mg en la planta, lo que aumenta el riesgo de deficiencia de Mg [10]. La deficiencia de Mg a largo plazo genera la acumulación excesiva de almidón y clorosis en varias especies de plantas, reduciendo aún más la tasa de fotosíntesis; por lo cual, se afecta el crecimiento de las plantas [11].

El Mg actúa de manera significativa en el crecimiento y desarrollo de las plantas, ya que implica 1) la estabilización de la estructura de la clorofila y los cloroplastos, aceptando la energía luminosa, transfiriéndola al aceptor, la fotosíntesis, la fijación y el metabolismo del carbono; 2) la carga de azúcar en el floema, otros nutrientes, y su transporte; 3 ) la regulación del equilibrio catión-anión, y la turgencia junto con el $\mathrm{K}$ en la vacuola, y 4) el mantenimiento de la estabilización y sintesis de macromoléculas. El desequilibrio de la homeostasis del Mg en las células afecta esos procesos [12].

En la actualidad se realizan prácticas agronómicas conducentes a disminuir la cantidad de aplicaciones de agroquímicos (cirugía, deshoje, fertilización, manejo de los residuos eliminados de la planta, entre otros). Por lo antes expuesto, en el presente trabajo se evaluó la severidad de Sigatoka negra (Mycosphaerella fijiensis Morelet) en plátano "Barraganete" bajo fertilización con magnesio.

\section{Materiales y métodos}

La investigación se condujo en la granja experimental Río Suma, Universidad Laica "Eloy Alfaro" de Manabí, extensión El Carmen, ubicada en la provincia de Manabí, cantón El Carmen, coordenadas DMS 0¹5’34,2" S, $79^{\circ} 25^{\prime} 39,2^{\prime \prime} 0$, con clima tropical húmedo, altitud de $263 \mathrm{msnm}$, temperatura media de $24,15^{\circ} \mathrm{C}$, precipitación de $2.806 \mathrm{~mm} /$ año, $86 \%$ de humedad relativa, heliofanía de 1.026 horas/luz/año y evaporación de $1.064 \mathrm{~mm} /$ año [13]. El análisis de suelo presentó niveles bajos de $\mathrm{NH}_{4}^{+}(11,61 \mathrm{ppm}), \mathrm{P}(4,56 \mathrm{ppm})$ y $\mathrm{Mg}(0,90 \mathrm{meq} / 100 \mathrm{~g})$, $\mathrm{y}$ niveles altos de $\mathrm{K}(0,50 \mathrm{meq} / 100 \mathrm{~g})$, con textura franco arenosa (62\% de arena, $28 \%$ de limo y $10 \%$ de arcilla) [14].

\section{Ensayos y tratamientos}

Se evaluó una plantación de plátano "Barraganete" (Musa sp. AAB), de segundo ciclo, con distancia de siembra de 2,5 m entre hileras x 1,8 m 
entre plantas $\left(4,5 \mathrm{~m}^{2}\right)$, para un total de 2.222 plantas/ ha (alta densidad). El número total de plantas fue de 288 distribuidas en tres bloques. Se realizaron aplicaciones de fertilizante fraccionados en tres partes (N-P-K y MgO). El fraccionamiento de $P$ fue realizado considerando que Avellán-Vásquez et al. [14] señalaron que incrementa la eficiencia, ya que es un elemento con poco movimiento en el suelo, por lo que las raíces absorben el que se encuentra cercano a ellas. Al ser añadido al suelo se encuentra soluble y disponible, aunque es fijado de manera rápida y deja de estar aprovechable para la planta. Esa baja movilidad genera que permanezca en la capa superior del suelo, perdiéndose principalmente a través de escorrentía superficial y erosión.

Los tratamientos se dispusieron en un diseño experimental en bloques completamente al azar, con tres repeticiones. La variación de la severidad durante el período de estudio se analizó con el paquete estadístico Statistical Analysis System (SAS v.9.1.3, 2020) [15], mediante la metodología de medidas repetidas en el tiempo a través del procedimiento de modelo lineal mixto (MIXED), a fin de estudiar el comportamiento longitudinal de los seis tratamientos de $\mathrm{MgO}$, seleccionando los modelos polinomiales de cuarto grado que mejor explicaron el comportamiento de esta variable a lo largo del tiempo. Semanalmente se cuantificaron la temperatura ambiental (medida con un termómetro digital), humedad relativa (higrómetro digital) y la temperatura de las hojas evaluadas (medida con un termómetro de infrarrojo), con el fin de observar la influencia de estas variables climáticas con la severidad de la enfermedad.

La fertilización consistió en la aplicación de una dosis estándar de 100,40 y $150 \mathrm{Kg} /$ ha de $\mathrm{N}, \mathrm{P}_{2} \mathrm{O}_{5}$ y $\mathrm{K}_{2} \mathrm{O}$, complementada con los tratamientos de fertilización con $\mathrm{MgO}$ a seis niveles $(0,25,50,75,100$ y $125 \mathrm{Kg} / \mathrm{ha}$ de $\mathrm{MgO}$ ), fraccionados en tres partes iguales y aplicados al suelo cuando las plantas emitieron las hojas 12, 18 y 24 . Los fertilizantes comerciales utilizados fueron urea $(46 \%$ de $\mathrm{N}$ ), fosfato diamónico (DAP; $18 \%$ de $\mathrm{N}$ y $46 \%$ de $\mathrm{P}_{2} \mathrm{O}_{5}$ ), muriato de potasio $\left(60 \%\right.$ de $\left.\mathrm{K}_{2} \mathrm{O}\right)$ y óxido de magnesio (30\% de $\mathrm{MgO})$.

Semanalmente se realizaron supervisiones de campo, labores de cirugía y deshoje; además de utilizar la escala de Fouré [16] para establecer la severidad de la enfermedad. Los síntomas de SN se reconocieron a través de siete estadios (Figura 1). Grados de infección: 1A. Pizca: pequeña mancha (punto) despigmentada de color blanco amarillento a marrón, visible solo en el envés (menor a cinco pizcas); 1B. Pizca: pequeña mancha (punto) despigmentada visible solo en el envés (mayor a cinco pizcas); 2C. Estría: raya alargada y ensanchada de color café oscuro a casi negro, visible en el haz de la hoja; 3C. Mancha: de forma elíptica, color café oscuro en el envés y negra en el haz de la hoja; 4C. Quema: mancha negra en el haz y envés, el centro se deprime y se rodea de un halo amarillo; 5C. 1/3 de la hoja quemada: similar a la anterior, ocupando una tercera parte de la hoja; y 6C. Más del 50\% de la hoja quemada: centro de la mancha se seca y necrotiza, adquiriendo un color grisáceo [16].
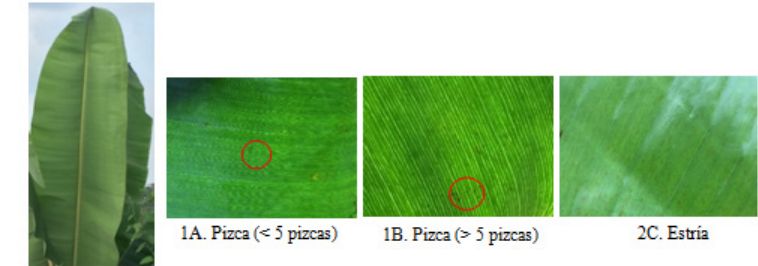

Sana

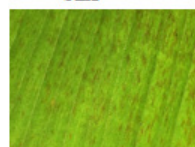

3C. Mancha
1A. Pizca $(<5$ pizcas $)$

1B. Pizca $(>5$ pizcas $)$

2C. Estría

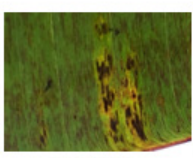

4C. Quema

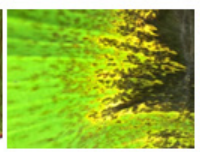

5C. $1 / 3$ de la hoja quemada hoja quemada

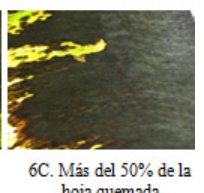

Figura 1. Grados de infección o estados de avance de la enfermedad causada por Sigatoka negra (Mycosphaerella fijiensis Morelet), en plátano "Barraganete", cultivado en la granja experimental Río Suma, Universidad Laica Eloy Alfaro de Manabí, Extensión El Carmen.

\section{Grado de severidad de la enfermedad}

Para estimar el grado de severidad de la enfermedad en las plantas en crecimiento (estado evolutivo), bajo tratamiento de fertilización, se tomó al azar una muestra de 10 plantas, en las cuales se ubicaron las hojas 3, 4 y 5 contando de arriba hacia abajo, pares e impares (Figura 2 ; [17]), y se determinó la existencia de algunos de los síntomas de acuerdo a la escala de Fouré [16] (Figura 1). Posteriormente se procedió al cálculo matemático que permitió determinar el grado de infestación en este tipo de plantas, clasificado como leve, alto o severo.

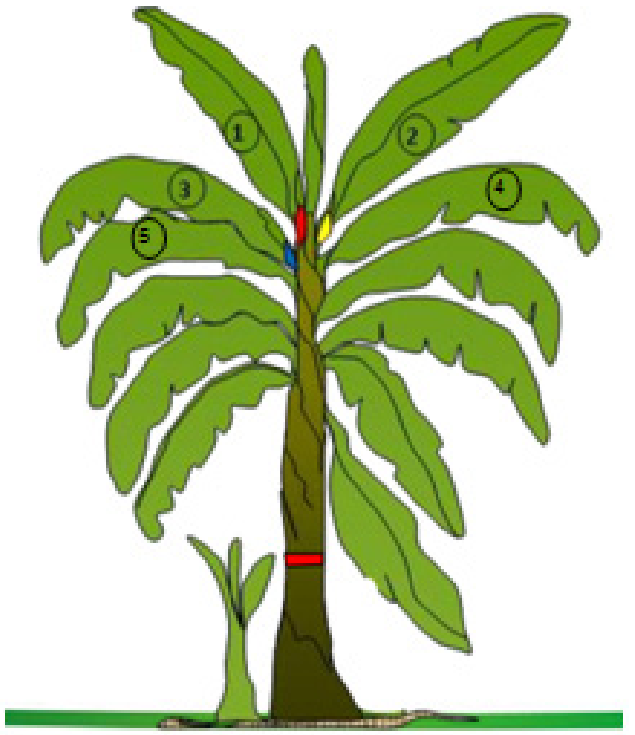

Figura 2. Esquema de posición y marcación de las hojas 1, 2, 3, 4 y 5 de una planta en estado evolutivo, para evaluar la incidencia o severidad de Sigatoka negra (Mycosphaerella fijiensis Morelet). Modificada de Calle y Yangali [17]. 
Se sumaron los valores de las hojas 3,4 y 5 de acuerdo al grado de infección (1A, 1B, 2C, 3C, 4C, 5C y 6C), obtenidos de la inspección realizada al cultivo semanalmente, para calcular la media con número de hojas $(\mathrm{MNH})(\mathrm{n}=10)$. Las letras equivalen a los valores de $A=1, B=2$ y $C=3$, los cuales se sumaron y se dividieron entre 10 para obtener la media (MLH). Posteriormente, se multiplicó MNH x MLH de las hojas 3, 4 y 5 . El resultado de la multiplicación anterior, se multiplicó una vez más por una constante, la cual varió de acuerdo al número de la hoja: para la hoja $3=120$, para la hoja $4=100$ y para la hoja $5=80$. Las constantes fueron obtenidas de acuerdo a los coeficientes arbitrarios de la severidad de la infección en las hojas 3, 4 y 5, como lo describió Orozco-Santo et al. [17] El resultado final indicó el grado de severidad de SN, considerando los siguientes niveles: cuando en la hoja 3 los valores fueron $<300$, recibieron la categoría de leve; en la hoja 4, cuando alcanzaron valores $>300$ hasta 500 recibieron la categoría de alto y cuando en la hoja 5 alcanzaron valores $>500$, la categoría asignada fue severa.

Se realizaron seis aplicaciones de fungicidas con productos de contacto y sistémicos, los ingredientes activos de estos productos fueron: mancozeb (100 g) + tebuconazole + triadimenol $(20 \mathrm{~mL})+$ mezcla de alquilaril y poliglicol 12,5\% (dos aplicaciones, intervalo de ocho días); difenoconazole (20 mL) + propineb (100 g) + mezcla de alquilaril y poliglicol 12,5\% (dos aplicaciones, intervalo 15 días) y carbendazim $(20 \mathrm{~mL})+$ mancozeb $(100 \mathrm{~g})+$ mezcla de alquilaril y poliglicol 12,5\% (dos aplicaciones, intervalo 15 días).

\section{Resultados y discusión}

La implementación de programas de manejo eficientes requiere disponer de tres tipos de información básica: 1) climática: particularmente cantidad y frecuencia de la lluvia y duración de la humedad en las hojas, ello permite valorar la evolución epidemiológica futura de la enfermedad [18, 19]; 2) biológicas: velocidad o ritmo de emisión de las hojas, hojas más jóvenes con síntomas y manchas necróticas; severidad en la floración de las 7 a las 9 semanas de después de la emisión del racimo [8] y la velocidad de la evolución de la enfermedad [6] y; 3) sensibilidad de las poblaciones a los principales fungicidas utilizados [21].

Dadas estas premisas, en las inspecciones de campo se cuantificaron variables ambientales al inicio y final de las evaluaciones para conocer las condiciones ambientales al momento de realizar los muestreos. La humedad relativa media fue de 85,60 y $85,40 \%$, respectivamente. La temperatura ambiental media fue de 24,62 y $25,32{ }^{\circ} \mathrm{C}$, respectivamente. La temperatura de la hoja 3 fue de $23,98^{\circ} \mathrm{C}$, para la hoja 4 de $23,88^{\circ} \mathrm{C}$ y para la hoja 5 de $23,84^{\circ} \mathrm{C}$. La diferencia térmica entre la hoja 3 y la 4 fue de $0,10^{\circ} \mathrm{C}$; entre la hoja 3 y la 5 fue de $0,14^{\circ} \mathrm{C}$ y entre la hoja 4 y la 5 fue de $0,04{ }^{\circ} \mathrm{C}$.

La precipitación media para el periodo evaluado fue de $40,14 \mathrm{~mm} / \mathrm{semana}$ y de $160,55 \mathrm{~mm} / \mathrm{mes}$, con una precipitación mínima de 0,00 mm y una máxima de 279,10 $\mathrm{mm}$; no obstante, durante el periodo de evaluación (cuatro meses), la precipitación total fue de 642,20 mm. Todos estos factores ambientales tuvieron una importancia relativa en la incidencia, severidad y diseminación de SN.

Álvarez et al. [22] señalaron que las precipitaciones elevadas y constantes, así como las temperaturas entre 26 y $28^{\circ} \mathrm{C}$, poseen un marcado efecto sobre los procesos de infección, germinación, penetración del patógeno y liberación del inóculo, mientras que Calle y Yangali [17] indicaron que el viento favoreció la diseminación. Igualmente, el hombre forma parte del patosistema al tomar decisiones sobre el uso de determinado cultivar, sistema de producción, manejo del hospedero y métodos de control de SN, entre otros [18].

Calle y Yangali [17] reportaron que el hongo se desarrolla bajo las condiciones de alta humedad y temperatura que se presentan en Ecuador durante la estación lluviosa (diciembre-mayo), y las estructuras reproductivas del hongo se activan a partir de $21^{\circ} \mathrm{C}$, para lo cual se requiere de la presencia de cierta humedad y/o película de agua sobre la hoja. A su vez, la maduración de las estructuras reproductivas provoca el desprendimiento de ascosporas y conidias, que diseminan la enfermedad, entonces las esporas pueden ser conducidas por el aire o salpique a las hojas vecinas, posándose sobre el tejido foliar nuevo, para iniciar un nuevo periodo de infección.

\section{Número de hojas}

El número de hojas por planta, de acuerdo con los tratamientos de fertilización, no presentaron una tendencia en particular; su comportamiento fue muy variable durante el tiempo de evaluación; no obstante, los tratamientos de 75 y $100 \mathrm{Kg} / \mathrm{ha}$ de $\mathrm{MgO}$ fueron los que mantuvieron una tendencia a incrementar el número de hojas por planta a partir de la semana 25 de evaluación, tal como puede apreciarse en la Figura 3. Para la semana 29, T5 presentó una media de 7,36 hojas/planta. En este sentido, el número de hojas que presenta la planta al momento de la emisión de la inflorescencia y desarrollo del racimo, es importante para garantizar la productividad y calidad de los frutos.

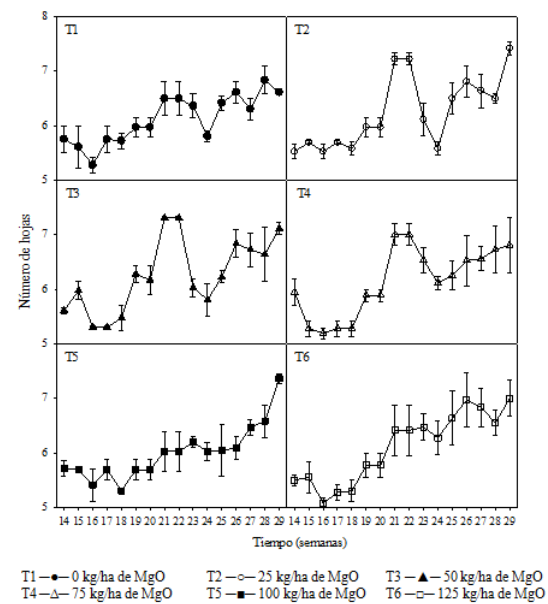

Figura 3. Número de hojas de plantas de plátano "Barraganete" fertilizadas con diferentes dosis de MgO, cultivadas en la granja experimental Río Suma, Universidad Laica Eloy Alfaro de Manabí, Extensión El Carmen. 
Se destaca también que en los tratamientos de 25,50 y $75 \mathrm{Kg} /$ ha de $\mathrm{MgO}$ en las semanas 21 y 22 se presentó el mayor número de hojas con una media de 7,17 hojas/planta y en la semana 29 de 7,11 hojas/plantas. Por otro lado, en la semana 16 todos los tratamientos mostraron el menor número de hojas. Posteriormente en la semana 24 los tratamientos de 0,25 y $50 \mathrm{Kg} / \mathrm{ha}$ de $\mathrm{MgO}$ presentaron una disminución del número de hojas (media 5,73 hojas/planta); resultante posiblemente del deshoje y cirugía debido a las altas precipitaciones que se presentaron en la semana 15 , y de la alta severidad de la enfermedad en las hojas 4 y 5 en la semana 24 .

Por otro lado, hubo diferencias significativas $(\mathrm{p}<0,01)$ para los muestreos realizados en las semanas 21, 22 y 29. En las semanas 21 y 22, se presentaron diferencias entre los tratamientos 50 (con 7,31 hojas), 25 y $75 \mathrm{Kg} / \mathrm{ha}$ de $\mathrm{MgO}$, al compararlos con los tratamientos de 125, 100 y 0 (con 6,08 hojas) Kg/ha de MgO. Igualmente, en la semana 29 se encontraron diferencias entre los tratamientos de 25 (con 7,42 hojas), 50, 100 y $125 \mathrm{Kg} / \mathrm{ha}$ de $\mathrm{MgO}$ al compararlos con los tratamientos de 75 y 0 (con 6,08 hojas) $\mathrm{Kg} / \mathrm{ha}$ de MgO.

En Urabá, Colombia, durante la fase vegetativa, la planta emitió entre 35 y 36 hojas, con una frecuencia de 1 hoja/semana en época de lluvias y entre 0,4 y 0,6 hoja/semana en condiciones de sequía [23]. Esta tasa de producción le permitió a la planta reemplazar las hojas que han cumplido su ciclo o que han sido afectadas por enfermedades tales como SN o por daños mecánicos. En total, de acuerdo con Turner et al. [24], la planta generó de 30 a 50 o más hojas en el ciclo de cultivo, pero solo mantuvo al mismo tiempo de 10 a 14 hojas fotosintéticamente activas, con lo cual garantizó altos rendimientos.

En plátanos del tipo Falso Cuerno cv. Dominico Hartón, cultivados entre 1.300 y 1.600 msnm, la transición floral sucedió alrededor de los cinco o seis meses de iniciado el proceso de producción de hojas con la emisión del $50 \%$ del total de las mismas, alrededor de las 19 hojas [25].

Aristizábal y Jaramillo [25] indicaron que para el mismo cultivo sembrado a $1.050 \mathrm{msnm}$, dicha etapa ocurrió cuando la planta había emitido 27 hojas. Por su parte, Hernández et al. [26] en Venezuela, ubicaron la transición floral en plátanos (cv. Hartón Enano) del mismo tipo (Falso Cuerno) en la hoja 25 (33\% de las plantas), en la hoja 27 (60\% de las plantas) y en la hoja 30 (80-100\% de las plantas).

\section{Severidad de la enfermedad}

En la Figura 4 se presenta la evolución de SN durante el tiempo de evaluación, cuyo comportamiento siguió un modelo polinomial de cuarto grado $(\mathrm{Y}=\mathrm{a}+\mathrm{bx}+$ $\left.\mathrm{cx}^{2}+\mathrm{dx}^{3}+\mathrm{ex}^{4}\right)$. En la misma se aprecia que en las semanas 14 y 15 se presentaron las mayores precipitaciones $(168,9$ y 279,1 mm, respectivamente), coincidiendo estas con niveles altos de incidencia (100\% durante todo el periodo de evaluación) y severidad para los seis tratamientos de fertilización con MgO. Sin embargo, los tratamientos de 50, 75 y $125 \mathrm{Kg} / \mathrm{ha}$ de $\mathrm{MgO}$ al inicio del ensayo (semana 14), presentaron la menor severidad en la hoja 3 con valores cercanos o inferiores a 300; los tratamientos testigo (0), 25 y $50 \mathrm{Kg} / \mathrm{ha}$ de $\mathrm{MgO}$ presentaron una severidad de la enfermedad superior al nivel leve (300). Esto conllevó a realizar aplicaciones de fungidas de contacto (protectante) y curativos (sistémicos), además de la realización de deshoje y cirugía (Figura 4).

En la semana 20 se presentaron los mayores valores de severidad de la enfermedad en la hoja 3 para todos los tratamientos de fertilización, superando inclusive el nivel severo $(>500)$ de SN. Esto se debió a dificultades de realizar cirugía y deshoje durante la semana 19, aunado a que en la semana 20 se presentaron precipitaciones que acumularon $44 \mathrm{~mm}$, y la presencia de altas temperaturas. Igualmente, en la semana 26 , los tratamientos de 75 y $100 \mathrm{Kg} / \mathrm{ha}$ de $\mathrm{MgO}$ alcanzaron un nivel alto de severidad (Figura 4).

Con el deshoje y cirugía, a intervalos semanales, se logró reducir la severidad de la enfermedad. Otras prácticas adicionales al deshoje sanitario también podrían ayudar en su manejo, como el apilamiento o acordonamiento del tejido enfermo en el suelo y la aplicación de urea al 10\% como inhibidor de la esporulación [27].

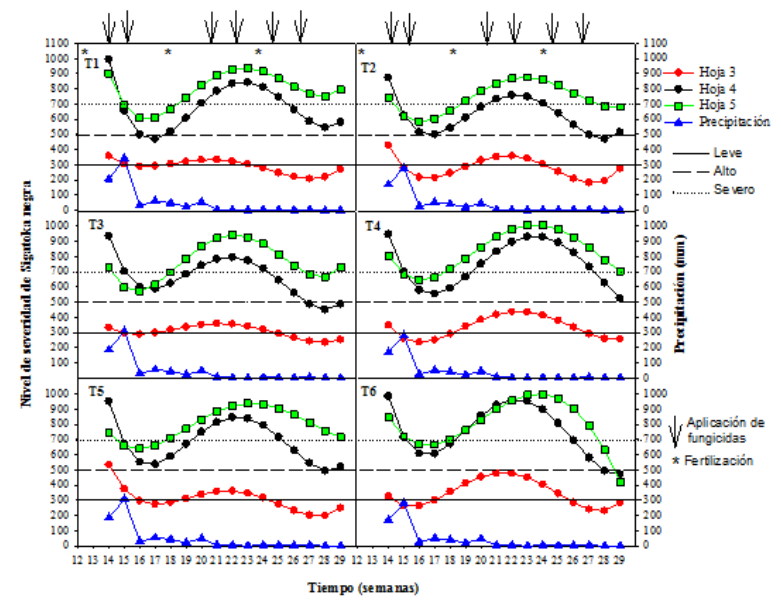

Figura 4. Severidad de Sigatoka negra (Mycosphaerella fijiensis Morelet), en plátano "Barraganete", cultivado en la granja experimental Río Suma, Universidad Laica Eloy Alfaro de Manabí, Extensión El Carmen, fertilizado con diferentes dosis de MgO. La primera aplicación de fertilizante se realizó en la semana 12.

Guzmán y Villalta [28] confirmaron la importancia del control de malezas en forma regular (herbicida o chapea), para un adecuado combate de la 
enfermedad y la posibilidad de utilizar coberturas vivas de suelo de porte bajo, sin detrimento en el control de SN. La nutrición mineral balanceada es también un aspecto relevante. Algunos elementos como el silicio, cobre, calcio, boro y zinc, contribuyen a reducir la severidad de la enfermedad [28].

Al discriminar la severidad en la hoja 3 para cada uno de los tratamientos de fertilización se observó que las mayores incidencias de SN se presentaron en la semana 14 en los testigos (0), 25, 75 y $100 \mathrm{Kg} / \mathrm{ha}$ de $\mathrm{MgO}$; en la semana 15 en los tratamientos 50, 100 y $125 \mathrm{Kg} / \mathrm{ha}$ de MgO; en la semana 16 en los tratamientos de 50 y $100 \mathrm{Kg} /$ ha de MgO; en la semana 18 en el tratamiento de $125 \mathrm{Kg} /$ ha de Mg0; en la semana 20 y 21 en todos los tratamientos; en la semana 23 en los tratamientos de 50,75 y $125 \mathrm{Kg} / \mathrm{ha}$ de $\mathrm{MgO}$; en la semana 24 en el tratamiento de $125 \mathrm{Kg} / \mathrm{ha}$ de MgO; en la semana 25 en el tratamiento de $75 \mathrm{Kg} / \mathrm{ha}$ de Mg0 y en la semana 26 en todos los tratamientos, excepto en el tratamiento $25 \mathrm{Kg} /$ ha de MgO (Figura 4).

Al realizar el análisis de la varianza para la severidad de la enfermedad por muestreos, se encontraron diferencias significativas $(\mathrm{p}<0,01)$ para algunos de ellos, por efecto de los tratamientos, dependiendo de la hoja evaluada (3, 4 y 5). En la semana 14 se encontraron diferencias en la hoja 5 entre el testigo y los tratamientos donde se aplicó MgO. En la semana 18 se presentaron diferencias en la hoja 3 entre los tratamientos 125 (317,63; nivel de severidad alto) y $0 \mathrm{Kg} / \mathrm{ha}$ de $\mathrm{MgO}$, al compararlo con los tratamientos de 25 (156,67; nivel de severidad leve), 50, 75 y $100 \mathrm{Kg} /$ ha de Mg0 (Figura 4).

Se encontraron diferencias en las semanas 21 y 22 en la hoja 3 entre los tratamientos 50, 100 y $125 \mathrm{Kg} /$ ha de $\mathrm{MgO}$ con respecto a los tratamientos 0, 25 y $75 \mathrm{Kg} /$ ha de $\mathrm{MgO}$. En este mismo orden de ideas, se encontraron diferencias significativas $(\mathrm{p}<0,01)$ en la semana 25 para las hojas 3, 4 y 5 . En la hoja 3 las diferencias fueron entre los tratamientos 0,75 y $125 \mathrm{Kg} / \mathrm{ha}$ de $\mathrm{MgO}$ y los tratamientos 25, 50 y $100 \mathrm{Kg} / \mathrm{ha}$ de $\mathrm{MgO}$. En la hoja 4, las diferencias fueron entre el tratamiento $75 \mathrm{Kg} / \mathrm{ha}$ de $\mathrm{MgO}$ y los tratamientos de 0, 25, 50, 100 y $125 \mathrm{Kg} /$ ha de MgO; mientras que en la hoja 5 las diferencias fueron entre los tratamientos $0,50,75,100$ y $125 \mathrm{Kg} /$ ha de $\mathrm{MgO}$ y el tratamiento $25 \mathrm{Kg} /$ ha de Mg0 (Figura 4).

Se considera que el avance de la enfermedad fue muy rápido cuando la hoja 3 , que era la más joven, se encontraba enferma; por lo que, al ser leve el grado de infestación, este es relativamente bajo y puede ser controlado o manejado a través de la realización de prácticas culturales y de ser necesario, ante el incremento del daño, con el uso de productos fungicidas protectantes o sistémicos, coincidiendo esto con lo señalado por Muñoz y Vargas [29].

Con relación a las hojas 4 y 5 en general, a partir de la semana 21 los valores obtenidos estuvieron por encima de los niveles de incidencia alto ( $>300$ a 500) y severo( $>500$; Figura 4), aun cuando en campo las hojas no se apreciaban tan afectadas. Estos hallazgos sugieren hacer algunas reflexiones de la situación de El Carmen, Ecuador, con relación a las condiciones agroecológicas y de manejo, lo cual conllevaría a profundizar investigaciones donde los valores de las constantes que se utilizaron para determinar la severidad de la enfermedad en esta zona sean ajustados de acuerdo a los escenarios ambientales imperantes.

Esto generó que se hicieran seis aplicaciones de fungicidas durante todo el periodo evaluado, las cuales se programaron una vez realizadas las inspecciones de campo y determinada la incidencia y severidad de la enfermedad, en las semanas 14 y 15 (mancozeb (100 g) + tebuconazole + triadimenol $(20 \mathrm{~mL})+$ mezcla de alquilaril y poliglicol 12,5\% (dos aplicaciones, intervalo de ocho días), en las semanas 20 y 22 (difenoconazole $(20 \mathrm{~mL}$ ) + propineb $(100 \mathrm{~g})+$ mezcla de alquilaril y poliglicol $12,5 \%$ (dos aplicaciones, intervalo 15 días) y en las semanas 24 y 26 (carbendazim $(20 \mathrm{~mL})+$ mancozeb $(100 \mathrm{~g})+$ mezcla de alquilaril y poliglicol 12,5\% (dos aplicaciones, intervalo 15 días), cirugía y deshoje semanal (Figura 4). Por otro lado, el programa de aplicación de fungicidas preventivos y curativos para SN, se debe diseñar contemplando los diferentes modos de acción de los ingredientes activos de los fungicidas, para disminuir los riesgos de resistencia del hongo a las moléculas.

Lo anterior concuerda con lo expuesto por Cervantes et al. [21], quienes reportaron que el manejo químico de SN se ha llevado a cabo con el uso de fungicidas protectores y sistémicos en suspensión acuosa, en emulsiones de aceite y agua, o en mezcla directamente con aceite mineral solo, con activadores de mecanismos de resistencia del hospedante, y últimamente mediante el uso de compuestos relacionados con la nutrición, tanto de origen químico como naturales. No obstante, el solapamiento de los productos aplicados con presencia de aceite mineral, sugiere un impedimento para la penetración de la luz solar a las hojas, lo cual afecta el contenido de clorofila, la fotosíntesis y por ende los rendimientos del cultivo.

Con esta metodología se trató de determinar la detección temprana de los síntomas de SN en las hojas 3, 4 y 5 ; sin embargo, para poder establecer la severidad se requiere de una gran precisión en el reconocimiento de la sintomatología de la enfermedad, este conocimiento permite establecer la frecuencia y el nivel de manejo cultural y con fungicidas tanto de acción protectante como sistémica; lo cual permite tener valores indicativos del nivel de daño presente en la plantación. Pérez [30] indicó que el uso de fungicidas para la protección contra la enfermedad recibe una atención importante, porque en áreas con una adecuada pluviometría para la producción bananera de clones susceptibles sino se aplican controles químicos, no se logra alcanzar un control satisfactorio de la enfermedad. 
En este sentido, se ha señalado que a través de la fertilización se favorece nutricionalmente la presencia de poblaciones epifitas de bacterias quitinolíticas y glucanolíticas, que presentan capacidad biorreguladora sobre el patógeno, y que se encuentran de manera natural en los ecosistemas de las plantas cultivadas [31]. No obstante, en la época lluviosa se presentó una menor disponibilidad de macronutrientes como sodio, magnesio y amonio; además, del contenido proteico que favorezca a la microbiota epifita [32].

Esta estrategia de control busca reducir el inóculo del patógeno, antes que las condiciones ambientales favorezcan su diseminación y establecimiento. Por lo tanto, es importante alcanzar su máxima eficacia en momentos de baja presión de la enfermedad como la época seca, y así lograr establecer poblaciones más abundantes y efectivas de los microbiotas antagonistas en las épocas lluviosas donde, la presión de la enfermedad es más fuerte [30].

\section{Conclusiones}

Las ecuaciones de los modelos polinómicos determinan que la menor incidencia de la enfermedad, se presenta con la menor dosis de $\mathrm{Mg}$ de $25 \mathrm{Kg} / \mathrm{ha}$ de $\mathrm{MgO}$, quizás debido a la movilización de $\mathrm{Mg}$ a las vacuolas para actuar en el mantenimiento del potencial osmótico, así como también para almacenar el Mg que se encuentra en exceso dentro de las plantas.

El manejo de Sigatoka negra debe seguir enfocado en la integración de procedimientos culturales y químicos. Los cambios en la percepción de los consumidores de productos más sanos y en la preocupación pública por detener la contaminación ambiental, tendrán una influencia marcada en las tecnologías de control que se implementan. En este sentido, hay que considerar los diferentes escenarios de producción de musáceas, por un lado, la utilización de materiales, cultivares o variedades menos susceptibles a la enfermedad especialmente en zonas de alta pluviometría; y en segundo lugar, la necesidad de realizar actividades de promoción de esos materiales, cultivares o variedades a nivel de consumidores, resaltando las bondades en calidad, menor contaminación, beneficios para la salud, entre otros, en mejora de la calidad de vida de la población.

\section{Referencias bibliográficas}

[1] Soorianathasundaram, K., Narayana, C.K. and Paliyath, G.: Bananas and Plantains. Encyclopedia of Food and Health.Oxford: Academic Press, 320327 (2016).

[2] Barrera V., J., Barraza A., F. y Campo A., R.: Efecto del sombrío sobre la Sigatoka negra (Mycosphaerella fijiensis Morelet) en cultivo de plátano cv Hartón (Musa AAB Simmonds). Rev. U.D.C.A Act. \& Div. Cient. Vol. 19, $\mathrm{N}^{\circ} 2$ (2016) 317-323.
[3] Hidalgo, M., Tapia, A., Rodríguez, W. y Serrano, E.: Efecto de la Sigatoka negra (Mycosphaerella fijiensis) sobre la fotosíntesis y transpiración foliar del banano (Musa sp. AAA, cv. Valery). Agron. Costarricense. Vol. 30, N 1 (2006) 35-41.

[4] Rodríguez, P. y Cayón, G.: Efecto de Mycosphaerella fijiensis sobre la fisiología de la hoja de banano. Agr. Col. Vol. 26, $\mathrm{N}^{\circ} 2$ (2008) 256-265.

[5] De Bellaire, L., Fouré, E., Abadie, C. y Carlier, J.: Black leaf streak disease is challenging the banana industry. Fruits. Vol. 65, Nº 6 (2010) 327-342.

[6] Etebu, E. y Young, W.: Control of black sigatoka disease: challenges and prospects. Afr. J. Agr. Res. Vol. 6, $\mathrm{N}^{\circ}$ (3) (2011) 508-514.

[7] Guzmán, M. y Villalta, R.: Efecto del método de control de malezas sobre el desarrollo de la Sigatoka negra y la producción del banano (Musa AAA cv. Grande naine). CORBANA. Vol. 33, N 60 (2007) 50-52.

[8] Barrera, J., Cayón, G. y Robles, J.: Influencia de la exposición de las hojas y el epicarpio de frutos sobre el desarrollo y la calidad del racimo de plátano "Hartón" (Musa AAB Simmonds). Agr. Col. Vol. 27, N 12009 73-79.

[9] Chen, Z.C., Peng, W.T., Li, J. and Liao, H. Functional dissection and transport mechanism of magnesium in plants. Seminars in Cell \& Developmental Biology. Vol. 74 (2018) 142-152.

[10] Medina Jiménez, F.: Nutrición magnésica de la platanera. Granja. Vol. 13 (2016) 22-23.

[11] Jin, X. L., Ma, C. L., Yang, L. T. and Chen, L. S.: Alterations of physiology and gene expression due to long-term magnesium-deficiency differ between leaves and roots of Citrus reticulata. J. Plant Physiol. Vol. 198 (2016) 103-115.

[12] Guo, W.: Magnesium homeostasis mechanisms and magnesium use efficiency in plants. pp. 197-213. In: Plant Macronutrient Use Efficiency. Molecular and Genomic Perspectives in Crop Plants.M.A. Hossain, T.Kamiya, D.J. Burritt, L.-S. P. Tran and T. Fujiwara (Eds.). Elsevier. AcademicPress. (2018).

[13] Instituto Nacional de Meteorología e Hidrología (INAMHI).: Anuario meteorológico. $\mathrm{N}^{\circ}$ 53-2013. Quito, Ecuador. Recuperado de http://www. serviciometeorologico.gob.ec/docum_institucion/ anuarios/meteorologicos/Am_2013.pdf. (2017).

[14] Avellán Vásquez, L., Cobeña Loor, N., Estévez Chica, S., Zamora Macías, P., Vivas Cedeño, J., González Ramírez, I. y Sánchez-Urdaneta, A.B.: Exportación y eficiencia del uso de fósforo en 
plátano 'Barraganete' (Musa paradisiaca L.). RevistaFitotecnia Mexicana. Vol. 43, N 1 (2020) 25-33.

[15] Statistical Analysis System (SAS). 2020. SAS STUDIO user's guide: Statistics. Versión 15.1. SAS Institute Inc., Cary, NC USA. https://support.sas. com/documentation/onlinedoc/stat/index proc. html\#stat151

[16] Fouré, E.: Les Cercosporiosis des bananiers et des plantainsauCameroun, Mycosphaerella fijiensis et M. musicola: amélioration des stratégies de lutteintégree par des étudesépidémiologiques et la luttegénétique. Centre RégionalBananiers et Plantains. Cameroun. In: Biological and Integrated Control of Highland Banana and Plantain Pests and Diseases. Gold and Gemmill Ed. Cotonou, Benin. (1991), 290-304.

[17] Calle, H. y Yangali, J.: La Sigatoka Negra en el Ecuador. En: I Seminario Internacional Metodología para la evaluación de prueba de eficacia para plaguicidas en los principales cultivos del Ecuador. SAGAT. Recuperado de http://www. agrocalidad.gob.ec/wp-content/uploads/2014/ 12/PresentacionSigatokaH-Calle-JYangali.pdf. (2014).

[18] Orozco-Santos, M., García-Mariscal, K., ManzoSánchez, G., Guzmán-González, S., MartínezBolaños, L., Beltrán-García, M., Garrido-Ramírez, E., Torres-Amezcua, J.A. and Canto-Canché, B. La sigatoka negra y su manejo integrado en banano. Libro Técnico Núm. 1. SAGARPA, INIFAP, CIRPAC, Campo Experimental Tecomán. Tecomán, Colima, México. (2013), 152.

[19] Orozco-Santos, M., Orozco-Romero, J., PérezZamora, O., Manzo-Sánchez, G., Farías-Larios, J. y da Silva Moraes, W.: Prácticas culturales para el manejo de la Sigatoka negra en bananos y plátanos. Tropical Plant Pathology. Vol. 33, № 3 (2008) 189196.

[20] Sáez Sáez, V. y Puche, M.: Uso de índices climáticos para el estudio del hongo sigatoka negra (Mycosphaerella fijiensis Morelet) en cultivos de plátanos. Estación Chama, sur del lago de Maracaibo Terra Nueva Etapa. Vol. XIX, N ${ }^{\circ} 28$ (2003) 11-33.

[21] Cervantes Alava, A,R., Sánchez-Urdaneta, A.B. y Colmenares de Ortega, C.B.: Efecto de las aplicaciones de fungicidas comerciales sobre el contenido de clorofila en e l cultivo de banano (Musa AAA). Agroecosistemas. Vol. 7, N 3 (2019) 45-49.

[22] Álvarez, E., Pantoja, A. Lederson Gañán y Ceballos, G.: La sigatoka negra en plátano y banano. Guía para el reconocimiento y manejo de la enfermedad, aplicado a la agricultura familiar. Organización de las Naciones Unidas para la Alimentación y la Agricultura (FAO). CIAT/FAO. (2013), 7.

[23] Mira, J., Díaz, A. y Hernández, M. : Influencia del régimen de lluvias sobre la productividad bananera de Urabá. En: Memorias. XXXIV Congreso Sociedad Colombiana de Control de Malezas y Fisiología Vegetal. COMALFI, Bogotá. (2004), 72.

[24] Turner, D.W., Fortescue, J.A. and Thomas, D.: Environmental physiology of the bananas (Musa spp.). Brazilian Journal of Plant Physiology. Vol. 19, $\mathrm{N}^{\circ} 4$ (2007) 463-484.

[25] Aristizábal, M. y Jaramillo, C.: Identificación y descripción de las etapas de crecimiento del plátano Dominico Hartón (Musa AAB). Agro. Vol. $18, \mathrm{~N}^{\circ} 1$ (2010) 29-40.

[26] Hernández, Y., Giménez, G. and Gómez, M.: Flowering locus $\mathrm{T}$ y Constan en Musa: Nuevos genes que participan en la transición floral en plátano Musa AAB cv. Hartón Enano. Rev. Fac. Agron. (LUZ). Vol. 27, ํㅜ 4 (2010) 524-554.

[27] Villalta, R. y Guzmán, M.: Capacidad de esporulación de Mycosphaerella fijiensis en tejido foliar de banano depositado en el suelo y efecto anti-esporulante de la urea. CORBANA. Vol. 31, $\mathrm{N}^{\circ}$ 58 (2005) 41-43.

[28] Azofeifa, D., Martínez, I., Furcal, P., Serrano, E. y Guzmán, M.: Efecto de la fertilización foliar con Ca, $\mathrm{Mg}$, Zn y B en la severidad de la Sigatoka negra y en el crecimiento y la producción del banano (Musa AAA, cv. Grande naine). CORBANA. Vol. 33, N 60 (2007) 41-44.

[29] Muñoz Ruiz, C. y Vargas Jarquín, E.: Manejo de Sigatoka negra (Mycosphaerella fijiensis var. difformis Morelet) en plátano cv. "Curarré" en San Carlos, Zona Norte. Tecnología en Marcha. Vol. 18, $\mathrm{N}^{\circ} 3$ (2005) 38-52.

[30] Pérez Vicente, L.: Manejo convencional y alternativo de la sigatoka negra en bananos: Estado actual y perspectivas. Fitosanidad. Vol. 10, N 1 (2006) 5572.

[31] Patiño H., L.F., Bustamante R. E. y Salazar P., L.M.: Efecto de sustratos foliares sobre la sigatoka negra (Mycosphaerella fijiensis Morelet) en banano (Musa $\times$ paradisiaca L.) y plátano (Musa acuminata Colla). Agricultura Técnica. Vol. 67, Nº 4 (2007) 437-445.

[31] Salazar, L.M., Patiño, L.F. y Bustamante, E.: Sustratos foliares para el incremento de bacterias quitinolíticas y glucanolíticas en la filosfera de banano. Rev. Facultad Nacional de Agronomía (Colombia).Vol. 59, № 2 (2006) 3449-3465. 


\section{REVISTA TECNICA}

DE LA FACULTAD DE INGENIERIA

UNIVERSIDAD DEL ZULIA

Vol. 44. N`1, Enero - Abril 2021, pp. 04 - 58

Esta revista fue editada en formato digital y publicada en Diciembre de 2020, por el Fondo Editorial Serbiluz, Universidad del Zulia. Maracaibo-Venezuela

www.luz.edu.ve

www.serbi.luz.edu.ve

www.produccioncientificaluz.org 